\title{
EXOhSPEC folded design optimization and performance estimation
}

Esther Lhospice ${ }^{\mathrm{a}, \mathrm{b}}$, Christophe Buisset ${ }^{*}$,, Hugh R.A. Jones ${ }^{\mathrm{c}}$, William E. Martin ${ }^{\mathrm{c}}$, Ronny Errmann ${ }^{\mathrm{c}}$, Sirinrat Sithajan ${ }^{\mathrm{a}}$, Chantira Boonsri ${ }^{\mathrm{a}}$, Piyamas Choochalerm ${ }^{\mathrm{c}}$, Guillem Anglada-Escude ${ }^{\mathrm{d}}$, David Campbell ${ }^{\mathrm{c}}$, Mary Angelie Alagao ${ }^{\mathrm{a}}$, Jitsupa Paenoi ${ }^{\mathrm{a}}$, Apirat Prasit ${ }^{\mathrm{a}}$, Thirasak Panyaphirawat ${ }^{\mathrm{a}}$, Somsawat Rattanasoon ${ }^{\mathrm{a}}$, Apichat Leckngam ${ }^{\mathrm{a}}$, David Mkrtichian ${ }^{\mathrm{a}}$, Suparerk Aukkaravittayapun ${ }^{\mathrm{a}}$

${ }^{a}$ National Astronomical Research Institute of Thailand (NARIT), 260 Moo 4, T. Donkaew, A. Maerim, Chiangmai, 50180, Thailand; 'bnstitut d'optique Graduate School, 2 Avenue Augustin Fresnel, 91120 Palaiseau, France; 'Centre for Astrophysics Research, University of Hertfordshire, Hatfield, AL10 9AB, UK; ' Queen Mary University of London, Mile End Road, London E1 4NS

\begin{abstract}
The EXOplanet high resolution SPECtrograph (EXOhSPEC) instrument is an echelle spectrograph dedicated to the detection of exoplanets by using the radial velocity method using $2 \mathrm{~m}$ class telescopes. This spectrograph is specified to provide spectra with a spectral resolution $\mathrm{R}>70,000$ over the spectral range from 400 to $700 \mathrm{~nm}$ and to reach a short-term radial velocity precision of $3 \mathrm{~m} / \mathrm{s}$. To achieve this the separation between two adjacent spectral orders is specified to be greater than 30 pixels and to enable a wide range of targets the throughput of the instrument is specified to be higher than $4 \%$. We present the results of the optimization of the spectrograph collimator performed and initial tests of its optical performance. First, we consider the spectrograph design and we estimate its theoretical performance. We show that the theoretical image quality is close to the diffraction limit. Second, we describe the method used to perform the tolerancing analyzes using ZEMAX software to estimate the optical performance of the instrument after manufacturing, assembly and alignment. We present the results of the performance budget and we show that the estimated image quality performance of EXOhSPEC are in line with the specifications. Third, we present the results of the stray light analysis and we show that the minimum ratio between the scientific signal and the stray light halo signal is close to 10,000. Finally, we provide a status on the progress of the EXOhSPEC project and we show the first results obtained with a preliminary version of the prototype.
\end{abstract}

Keywords: spectroscopy, optical design, tolerancing analyzes, Echelle spectrograph, astronomy.

\section{INTRODUCTION}

The EXOplanet high resolution SPECtrograph (EXOhSPEC) project aims at developing a new kind of compact and costeffective Echelle spectrographs dedicated to high resolution spectroscopy for $2 \mathrm{~m}$ diameter class telescopes. In the existing high resolution spectrographs already installed on large telescopes such as the High Accuracy Radial velocity Planet Searcher (HARPS) installed in 2003 at the ESO La Silla 3.6m telescope [1], the entrance face of the spectrometer multi-mode fiber is placed at the telescope image plane. The fiber core diameter is designed to fit the size of the spot image based on the seeing conditions and the telescope focal length. The fiber beam aperture is adjusted to be equal to the telescope beam aperture. The output face of the fiber is placed at the entrance of the spectrograph which volume is driven by the fiber core diameter and aperture number. In the case of the HARPS spectrograph whose spectral resolution is close to 100,000 , the volume of the vacuum vessel required to insulate the instrument from temperature and pressure variations is equal to $2 \mathrm{~m}^{3}$ [2].

*christophe@narit.or.th; phone +66 91497 9919; www.narit.or.th

The objective of the EXOhSPEC project is thus to reduce the overall volume and hence cost of the instrument. This might be achieved by the use of i) tapered fibers to reduce the diameter of the fiber connected to the spectrograph and/or 
ii) an adaptive optics to inject on a fiber of small core diameter a beam of light whose wavefront has been corrected for the distortions induced by atmospheric turbulence.

The specifications of the spectrograph are represented in Table 1. This spectrograph is specified to provide a spectral resolution higher than 100,000 over the spectral domain from 400 to $800 \mathrm{~nm}$. We specify the spectrograph to provide diffraction limited images over $90 \%$ of the specified wavelength range to make sure that we can reach the spectral resolution specification. We also specified the stability to be $3 \mathrm{~m} / \mathrm{s}$ over 20 minutes that will be reached by passive stabilization, fiber mode control and sub-pixel processing.

We specify the separation between two adjacent spectral orders to be larger than 30 pixels. This is to ensure that the spectrograph will be able to simultaneously record a scientific and spectral calibration spectrum. The objective of this specification is thus to guarantee that the scientific and the calibration spectra will not overlap and will be correctly extracted.

Since 2017, the University of Hertfordshire (UH) and NARIT have designed and developed a prototype to demonstrate the feasibility of the proposed concept and to test the various subsystems. We plan to test and validate on-sky this prototype by using the NARIT medium size telescopes and the $2.4 \mathrm{~m}$ Thai National Telescope (TNT) [4]. The prototype is specified to provide a spectrum higher than 70,000 over a spectral domain at least 400 to $700 \mathrm{~nm}$ and must be diffraction limited over $90 \%$ of this spectral interval. The distance between two adjacent spectral orders is equal to 30 pixels similarly to the specification of the final system. The throughput is specified to be higher than $4 \%$.

Table 1. EXOhSPEC specifications for the final system and for the prototype.

\begin{tabular}{|c|c|c|c|}
\hline Req. $\mathbf{n}^{\mathbf{0}}$ & Performance & Prototype & Final system \\
\hline 1 & Spectral domain & {$[400 \mathrm{~nm}, 700 \mathrm{~nm}]$} & $>100,000$ \\
\hline 2 & Spectral resolution & $>70,000$ & $\mathrm{~nm}, 1000 \mathrm{~nm}]$ \\
\hline 3 & Image quality & $\begin{array}{c}\text { Diffraction limited across } 90 \% \\
\text { of specified spectral domain }\end{array}$ & $\begin{array}{c}\text { Diffraction limited across } 90 \% \text { of } \\
\text { specified spectral domain }\end{array}$ \\
\hline 4 & $\begin{array}{c}\text { Distance between } \\
\text { adjacent orders }\end{array}$ & $>30$ pixels & $>30$ pixels \\
\hline 5 & Throughput & $>4 \%$ & $3 \mathrm{~m} / \mathrm{s}$ over 1 hour \\
\hline 6 & Stability & - & \\
\hline
\end{tabular}

In Section 2, we describe the spectrograph design and we present the theoretical performance. In particular, we show that the theoretical system is diffraction limited over the prototype specified spectral domain. In Section 3 we present the results of the tolerancing analysis performed to estimate the image quality variation due to the manufacturing, the assembly and the alignment errors. We estimate that the image quality in operational conditions should be close to the diffraction limit over the spectral domain from 450 to $700 \mathrm{~nm}$. In Section 4 we describe the assumptions and the results of the stray light analysis. We show that the ratio between the signal and the stray light halo should be close to 10,000 . Finally in Section 5, we describe the status of the project and we present the on-sky results we have obtained with a preliminary version of the prototype that includes only off-the-shelf components.

\section{SPECTROGRAPH PROTOTYPE DESIGN AND THEORETICAL PERFORMANCE}

\subsection{Design overview}

Figure 1 represents the schematic view of the full system that comprises the Injection Unit (IU), the Injection Fiber (IF), the Mode Scrambler (MS), the Calibration Lamp (CL), the Bifurcated Fiber (BF) and the Spectrograph Assembly (SA). The telescope collects and directs the light toward the IU. This unit includes one beam splitter that reflects the light toward one guiding camera and transmits the light toward the IF. This fiber directs the light toward the MS that aims at mitigating the impact of the fiber mechanical and thermal modifications on the mode patterns. The beam transmitted by 
the MS is injected on one input fiber of the BF toward the Spectrograph. The CL injects the light on the second input fiber of the BF. The spectrograph assembly comprises one collimator ("Triplet" lens), a cross-disperser prism, grating, folding mirror and a detector. The detector simultaneously records the spectrum from the scientific object and from the calibration lamp.

Figure 1. EXOhSPEC system overview including the injection unit, the mode scrambler, the spectrograph assembly and the connecting fibers.

The optical design of the spectrograph assembly is represented in Figure 2. The bifurcated fiber output is positioned slightly off-axis at the focal plane of the apochromatic triplet. This lens collimates and directs the beam toward the prism that achieves the spectral separation of the diffraction orders. The beam transmitted by the prism is incident on the grating which is the aperture stop of the spectrograph. This grating is placed in a Littrow configuration and reflects the beam toward the dispersive prism and the achromatic triplet lens. This triplet focuses the beam toward an off-axis folding mirror that reflects the beam toward the detector located in the image plane.

The bifurcated fiber is made of two fibers of core diameters equal to $10 \mu \mathrm{m}$ and of numerical aperture equal to 0.1 and aperture number $\mathrm{F}_{\text {Fiber }} \approx 5$. The apochromatic triplet has a focal $\mathrm{f}_{\mathrm{Col}}=150 \mathrm{~mm}$ and comprises three lenses L2, L2 and L3 whose properties are summarized in Table 2. The lenses L1 and L2 are both made of $N-F K 51 A$ glass since L3 is made of $N-K Z F S 11$. The dipsersive prism is made of $\mathrm{F} 2$ and the angle of this prism is equal to 50 degree. This value has been adjusted i) to reach the specified separation between the two adjacent spectral orders and ii) to image the specified spectraldomain on the detector.

The grating corresponds to the Thorlabs model GE2550-0363 Echelle that comprises 31.6 Grooves/mm and a Blaze angle of $63^{\circ}$. The mechanical dimensions are equal to $25 \mathrm{~mm}$ x $50 \mathrm{~mm}$ x $9.5 \mathrm{~mm}$ and the clear aperture corresponds to a surface of $22.5 \times 45.0 \mathrm{~mm}$ [5]. The EXOhSPEC aperture stop of diameter $\mathrm{D}_{\mathrm{AS}}=20 \mathrm{~mm}$ is located on the diffraction grating. The aperture number of EXOhSPEC is thus $\mathrm{F} \#_{\text {Spectro }}=\mathrm{f}_{\mathrm{Col}} / \mathrm{D}_{\mathrm{AS}} \approx 7$. The folding mirror is made of the aluminized hypotenuse of a right-angle prism of length equal to $20 \mathrm{~mm}$. In this model, the detector comprises $4 \mathrm{~K}$ x $4 \mathrm{~K}$ square pixels of length equal to $5 \mu \mathrm{m}$ thus covering a surface equal to $20 \times 20 \mathrm{~mm}^{2}$. The distance between the prism and the detector is equal to $20 \mathrm{~mm}$ along the optical axis that is considered as the minimum value to be compatible with a typical backfocus length for a commercial camera. During the optimization process, we adjusted the orientation of the detector around the $\mathrm{x}$ and the $\mathrm{y}$ axes to optimize the image quality. We found that the orientation of the detector that provides the best image quality corresponds to a rotation around the x-axis $\theta_{x d e t}=4.7^{\circ}$ and a rotation around the $\mathrm{y}$-axis $\theta_{y d e t}=-2.3^{\circ}$. 
Figure 2. EXOhSPEC prototype optical design based on our ZEMAX model.

Table 2. The apochromatic triplet lenses properties for the prototype

\begin{tabular}{|c|c|c|c|}
\hline Lens & Glass & Radius of curvature & Thickness \\
\hline L1 & N-FK51A & $58.774 \mathrm{~mm} ; 474.365 \mathrm{~mm}$ & $9.503 \mathrm{~mm}$ \\
\hline L2 & N-KZFS11 & $81.529 \mathrm{~mm} ; 41.224 \mathrm{~mm}$ & $5.893 \mathrm{~mm}$ \\
\hline L3 & N-FK51A & $59.604 \mathrm{~mm} ; 287.330 \mathrm{~mm}$ & $10 \mathrm{~mm}$ \\
\hline
\end{tabular}

\subsection{Theoretical geometrical performance}

The spot diagram of the theoretical system with perfect alignment and optical surfaces is represented in Figure 3 and the Fast Fourier Transform (FFT) Point Spread Functions (PSF) are represented in Figure 4. They show the spectral orders $\mathrm{m}=80,106,125$ and 141 with corresponding central wavelengths $\lambda_{\mathrm{c}, 80} \approx 451 \mathrm{~nm}, \lambda_{\mathrm{c}, 106} \approx 532 \mathrm{~nm}, \lambda_{\mathrm{c}, 125} \approx 532 \mathrm{~nm}$ and $\lambda_{\mathrm{c}, 141} \approx 400 \mathrm{~nm}$ thus covering the prototypes specified spectral domain.

We notice on the spot diagrams that the theoretical prototype is diffraction limited in the diffraction orders $\mathrm{m}=80,106$ and 125 covering the spectral domain from 447 to $710 \mathrm{~nm}$. We also notice that the spot image is slightly larger than the diffraction limit in the diffraction order $\mathrm{m}-141\left(\lambda_{\mathrm{c}, 141} \approx 400 \mathrm{~nm}\right)$. We conclude that the system is diffraction limited over the specified spectral domain [447 nm, $710 \mathrm{~nm}$ ] and slightly above the diffraction limit across 400 to $447 \mathrm{~nm}$. We thus deduce that the theoretical image quality of the prototype is compliant with our specification.

We have estimated that the spectrum image on the detector covers a surface equal to $12 \mathrm{~mm} \times 16 \mathrm{~mm}$ that is compatible with the detector surface of $20 \mathrm{~mm}$ x $20 \mathrm{~mm}$. We have also calculated the distance between two adjacent diffraction orders. The minimum distance is 32 pixels between the diffraction orders $\mathrm{m}=80$ and $\mathrm{m}=81$ of respective central wavelengths equal to $704.9 \mathrm{~nm}$ and $696.2 \mathrm{~nm}$. This distance increases when the wavelength decreases and is equal to 60 pixels between the diffraction orders 124 and 125 at wavelengths close to $450 \mathrm{~nm}$. We thus deduce that the prototype theoretical system is compliant to the specified geometrical performance in image quality, distance between adjacent spectral orders and spectrum image size of the detector. 


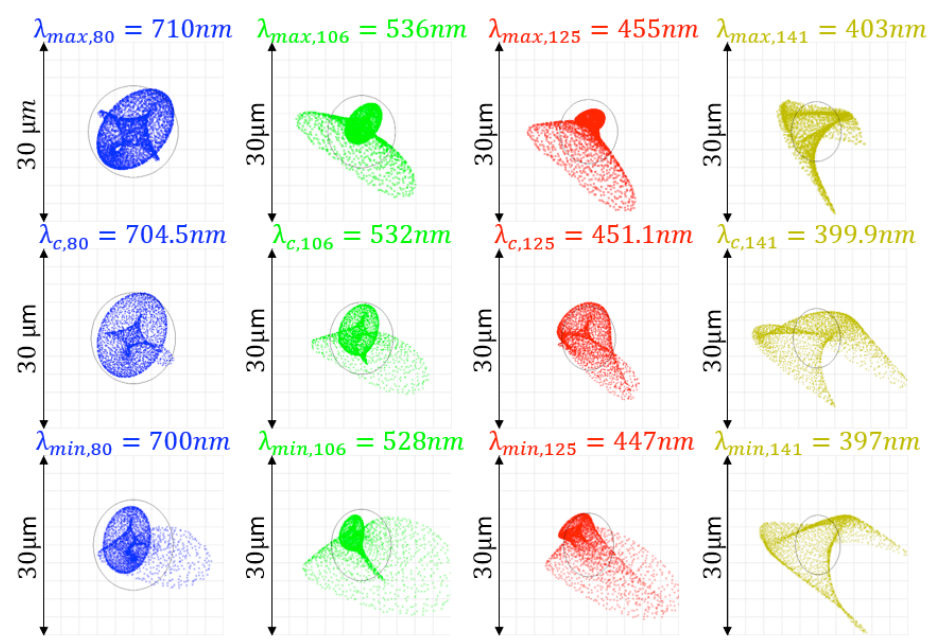

Figure 3. Spot diagram of the theoretical system for the diffraction orders $m=80,106,125$ and 141 for the respective central wavelengths $\lambda_{\mathrm{C}, 80}=704.5 \mathrm{~nm}, \lambda_{\mathrm{C}, 106}=532 \mathrm{~nm}, \lambda_{\mathrm{C}, 125}=451.1 \mathrm{~nm}$ and $\lambda_{\mathrm{C}, 141}=399.9 \mathrm{~nm}$ covering the specified spectral domain of the prototype.

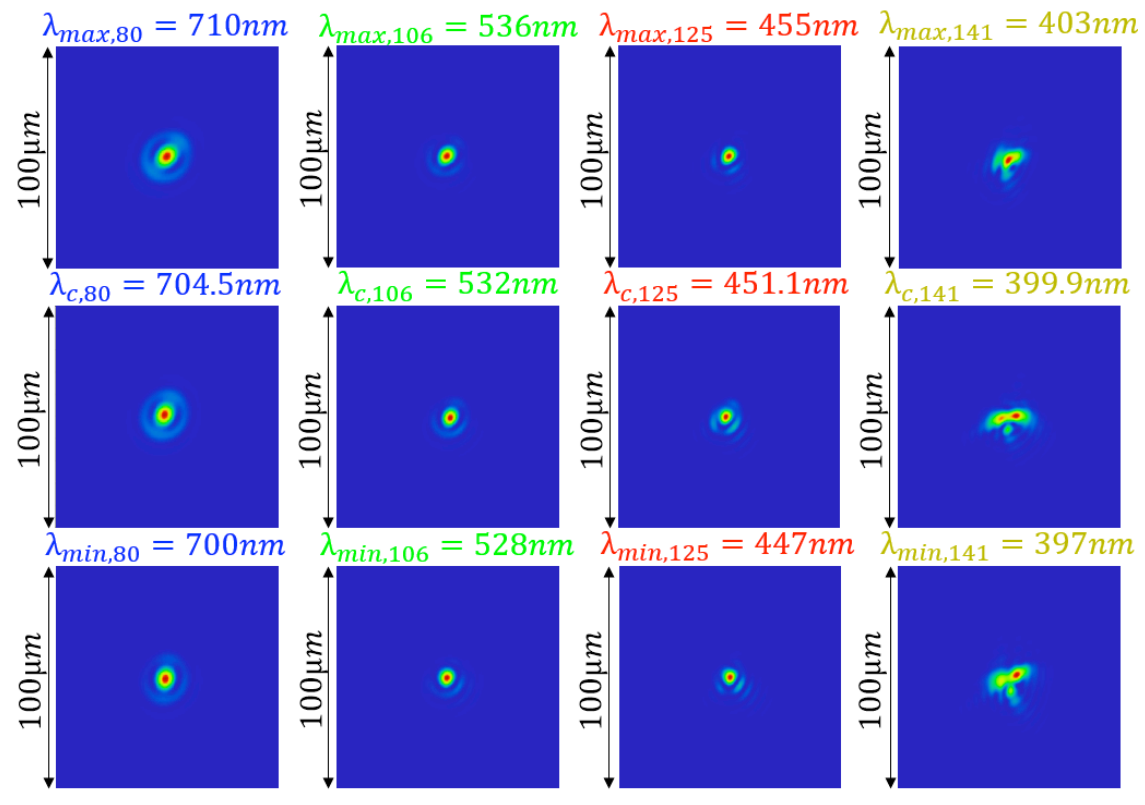

Figure 4. FFT PSF of the theoretical system for the diffraction orders $m=80,106,125$ and 141 for the respective central wavelengths $\lambda_{\mathrm{C}, 80}=704.5 \mathrm{~nm}, \lambda_{\mathrm{C}, 106}=532 \mathrm{~nm}, \lambda_{\mathrm{C}, 125}=451.1 \mathrm{~nm}$ and $\lambda_{\mathrm{C}, 141}=399.9 \mathrm{~nm}$ covering the specified spectral domain of the prototype.

\subsection{Throughput}

The theoretical transmission of the EXOhSPEC spectrometer assembly is calculated as follows

$$
T_{\text {Spectro }}=T_{\text {Col }}{ }^{2} \times T_{\text {Prism }}^{2} \times R_{\text {Grating }} \times R_{\text {Mirror }} \times T_{A M},
$$

where $T_{\mathrm{Col}}$ is the transmission of the collimator, $T_{\text {Prism }}$ is the transmission of the prism, $R_{\text {Grating }}$ the reflectivity of the grating (including the diffraction efficiency) and $\mathrm{T}_{\mathrm{AM}}$ is the equivalent throughput due to the aperture mismatch between the spectrometer and the bifurcated fiber. We estimate that $T_{\mathrm{Col}}=78 \%$ and $T_{\text {Prism }}=98 \%$ correspond to the transmission of the anti-reflective coatings of maximum reflectivity specified as $1 \%$. We assumed that the transmission of each glass 
is equal to 1 over the specified spectral domain. We estimate that $\mathrm{R}_{\text {Mirror }}=50 \%$ is the grating efficiency and that $\mathrm{R}_{\text {Mirror }}=$ $90 \%$ corresponds to the typical reflectivity of a surface coated with aluminum in the visible region..

The EXOHSPEC prototype aperture number $\mathrm{F}_{\text {Spectro }}=6.6$ is defined by the focal ratio of the collimator and the grating clear aperture while the fiber aperture number is $\mathrm{F} \#_{\text {Fiber }}=5$. There is thus a loss of light due to the aperture mismatch between the fiber and the spectrograph aperture numbers as represented in Figure 5. The throughput equivalent to this aperture mismatch is

$$
T_{A M}=\left(F \#_{F i b e r} / F \#_{C o l}\right)^{2} \approx 51 \% .
$$

We thus estimate that the throughput of the spectrograph assembly should be equal to $18 \%$. We deduce that the throughput of EXOhSPEC injection unit, injection fiber, mode scrambler and bifurcated fiber should be higher than $22 \%$ to comply with the specification on the spectrograph prototype: $T_{\text {Spectro }}>4 \%$.

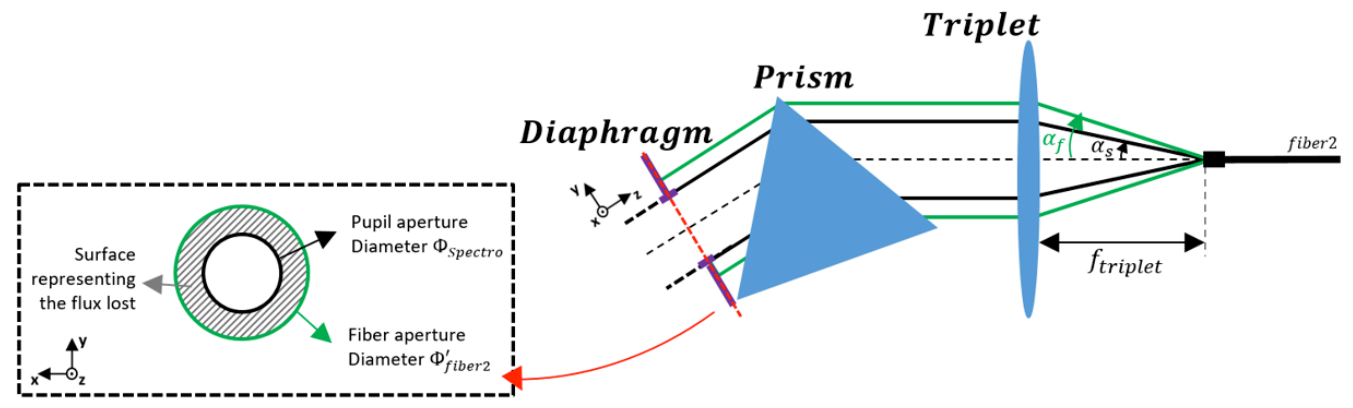

Figure 5. A schematic representation of the mismatch between the fiber and the spectrograph numerical apertures.

\section{IMAGE QUALITY TOLERANCING ANALYSES}

\subsection{Manufacturing errors}

Table 3 represents the assumptions used to perform the manufacturing error tolerancing analysis. This analysis aims at estimating the impact on the spot image due to manufacturing error on the optical elements. The assumptions on the lens triplet manufacturing errors are based on the manufacturing tolerancing chart of Optimax [6]. The maximum error on the index of refraction of L1, L2 and L3 glasses will be equal to 0.0005 while the error on the Abbe number will vary in the range $+/-5 \%$. We assume that the maximum surface irregularities will be equal to $200 \mathrm{~nm}$ Peak To Valley (PTV) and that the maximum error on the radius of curvatures will be equal to $+/-0.1 \%$. We assume that the maximum position and orientation errors of each optical surface will be equal to 1' and $50 \mu \mathrm{m}$ respectively. We assume that the maximum error on the prism will be equal to $5^{\prime}$ and that the maximum surface irregularities will be equal to $100 \mathrm{~nm}$ PTV. Finally, we assume that the maximum surface irregularities of the grating will be equal to $200 \mathrm{~nm}$ PTV.

Table 3. We summarise our assumptions of the manufacturing errors.

\begin{tabular}{|c|c|c|}
\hline Component & Parameter & Tolerance \\
\hline \multirow{4}{*}{$\begin{array}{c}\text { Apochromatic lenses } \\
\text { L1, L2 and L3 }\end{array}$} & Index of refraction & \pm 0.0005 \\
\cline { 2 - 3 } & Abbe number & $\pm 0.5 \%$ \\
\cline { 2 - 3 } & Surface irregularity & $\pm 200 \mu \mathrm{m}$ \\
\cline { 2 - 3 } & Radius of curvature & $\pm 0.1 \%$ \\
\cline { 2 - 3 } & Face tilts & \pm 1 \\
\cline { 2 - 3 } & Central Thickness & $\pm 0.05 \mathrm{~mm}$ \\
\hline
\end{tabular}




\begin{tabular}{|c|c|c|}
\hline \multirow{2}{*}{ Prism } & Prism angle & $\pm 5^{\prime}$ \\
\cline { 2 - 3 } & Surface irregularity & $\pm 100 \mathrm{~nm}$ \\
\hline Grating & Surface irregularity & $\pm 200 \mathrm{~nm}$ \\
\hline
\end{tabular}

The tolerancing analysis has been performed using a dedicated Monte-Carlo analysis within the ZEMAX Optics Studio software. This analysis comprised 1550 runs that correspond to approximatively 20 runs per tolerancing parameter. We used three compensators to optimize the image quality for each run: the fiber position along the optical axis, the detector focus and its tip-tilt. These compensators correspond to the degree of freedom that will be made during the spectrograph alignment.

In order to make sure that our tolerancing analysis cover the specified spectral domain, we have performed the tolerancing analysis at the following diffraction orders and wavelengths:

- $\mathrm{m}=141$ at the wavelengths $\lambda_{\min , 141}=397 \mathrm{~nm}, \lambda_{\mathrm{C}, 141}=400 \mathrm{~nm}$ and $\lambda_{\mathrm{Max}, 141}=403 \mathrm{~nm}$.

- $\mathrm{m}=125$ at the wavelengths $\lambda_{\min , 125}=447 \mathrm{~nm}, \lambda_{\mathrm{C}, 125}=451 \mathrm{~nm}$ and $\lambda_{\mathrm{Max}, 125}=455 \mathrm{~nm}$.

- $\mathrm{m}=106$ at the wavelengths $\lambda_{\min , 106}=528 \mathrm{~nm}, \lambda_{\mathrm{C}, 106}=532 \mathrm{~nm}$ and $\lambda_{\mathrm{Max}, 106}=536 \mathrm{~nm}$.

- $\mathrm{m}=80$ at the wavelengths $\lambda_{\min , 80}=700 \mathrm{~nm}, \lambda_{\mathrm{C}, 80}=705 \mathrm{~nm}$ and $\lambda_{\mathrm{Max}, 80}=710 \mathrm{~nm}$.

The results of the manufacturing errors tolerancing analysis show that the spot image radius maximum increase is reached for the diffraction order $m=125$. Figure 6 represents the cumulative probability curves of the RMS spot radius $\Delta \mathrm{R}_{\text {Manuf }}$ at the minimum, the central and the maximum wavelength of the diffraction order $\mathrm{m}=125$. We notice that in the realistic case ( $95 \%$ cumulative probability), the maximum value of $\Delta \mathrm{R}_{\text {Manuf }}$ is reached at the wavelength $\lambda_{\min , 125}=447 \mathrm{~nm}$ and is equal to $1.9 \mu \mathrm{m}$. The results of the tolerancing analysis also show that in the realistic case, the spot image radius enlargement is close to $1 \mu \mathrm{m}$.

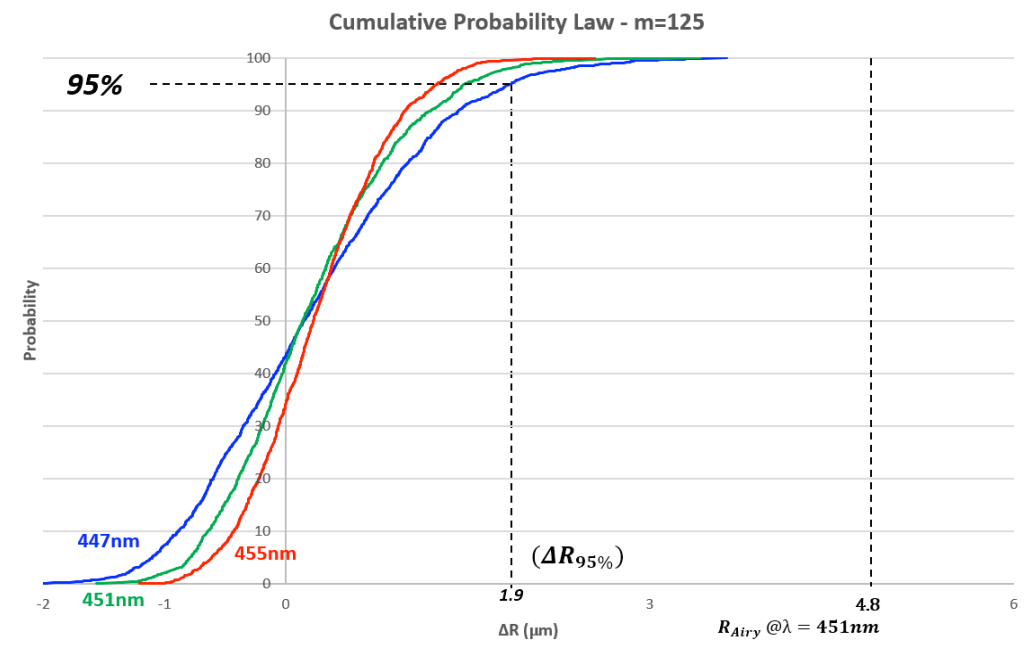

Figure 6. spot diagram RMS spot size cumulative probability after the manufacturing errors tolerancing analysis.

\subsection{Assembly errors}

The objective of the tolerance analysis dedicated to the assembly errors is to estimate the spot image enlargement due to the position and the orientation errors of each single lens of the collimator. These errors will be induced by the manufacturing error of the barrel manufactured at the NARIT mechanical workshop using a Computer Numerical Control machine. Based on our previous experience with the manufacturing of a lens barrel for the TNT focal reducer 
project [7], we assumed a maximum positioning error equal to $25 \mu \mathrm{m}$ and a maximum orientation error equal to 0.03 degree (2 arcminutes).

The tolerancing analysis has been performed by sorting randomly 400 EXOhSPEC optical systems in which i) the L1, $\mathrm{L} 2$ and $\mathrm{L} 3$ positions have been randomly changed along $\mathrm{X}, \mathrm{Y}$ and $\mathrm{Z}$ by $+/-25 \mu \mathrm{m}$ and ii) the orientations around the $\mathrm{X}$ and $\mathrm{Y}$ axes by $+/-0.03$ degrees (15 parameters in total). That corresponds to more than 25 Monte-Carlos runs for each degree of freedom. For each run we optimized three compensators: the fiber position along the optical axis, the detector focus and its tip-tilt.

Figure 8 represents the cumulative probability calculated at the diffraction order $\mathrm{m}=125$ at the wavelengths $\lambda_{\min , 125}=447 \mathrm{~nm}, \lambda_{C, 125}=451 \mathrm{~nm}$ and $\lambda_{\text {Max }, 125}=455 \mathrm{~nm}$. We notice that in the realistic case ( $95 \%$ cumulative probability), the maximum variation of the spot radius is $\Delta \mathrm{R}_{\text {Assy }}=2.9 \mu \mathrm{m}$.

The results obtained at the diffraction orders $\mathrm{m}=80,106,125$ and 141 show that $\Delta \mathrm{R}_{\text {Assy }}$ should vary between $1.5 \mu \mathrm{m}$ and $2.9 \mu \mathrm{m}$. The minimum values are obtained at the diffraction orders $\mathrm{m}=141$ and $\mathrm{m}=80$ where $\Delta \mathrm{R}_{\text {Assy }} \approx 1.5 \mu \mathrm{m}$. It is also important to mention that at the diffraction orders $\mathrm{m}=80$ and $141, \Delta \mathrm{R}_{95 \%}$ is quasi constant at the wavelengths $\lambda_{\min }, \lambda_{\mathrm{C}}$ and $\lambda_{\text {Max }}$. The maximum value of $\Delta \mathrm{R}_{\text {Assy }}$ is obtained at the diffraction order $\mathrm{m}=125$ and is equal to $2.9 \mu \mathrm{m}$ at the wavelength $\lambda_{\min , 125}=447 \mathrm{~nm}$.

Figure 7. Illustration of some of the potential assembly errors in the EXOhSPEC collimator. The top-panel shows the ideal theoretical optical system; where the bottom panel illustrates lens L1 decentered along the z-axis and lens L3 tilted around the $\mathrm{x}$-axis. 


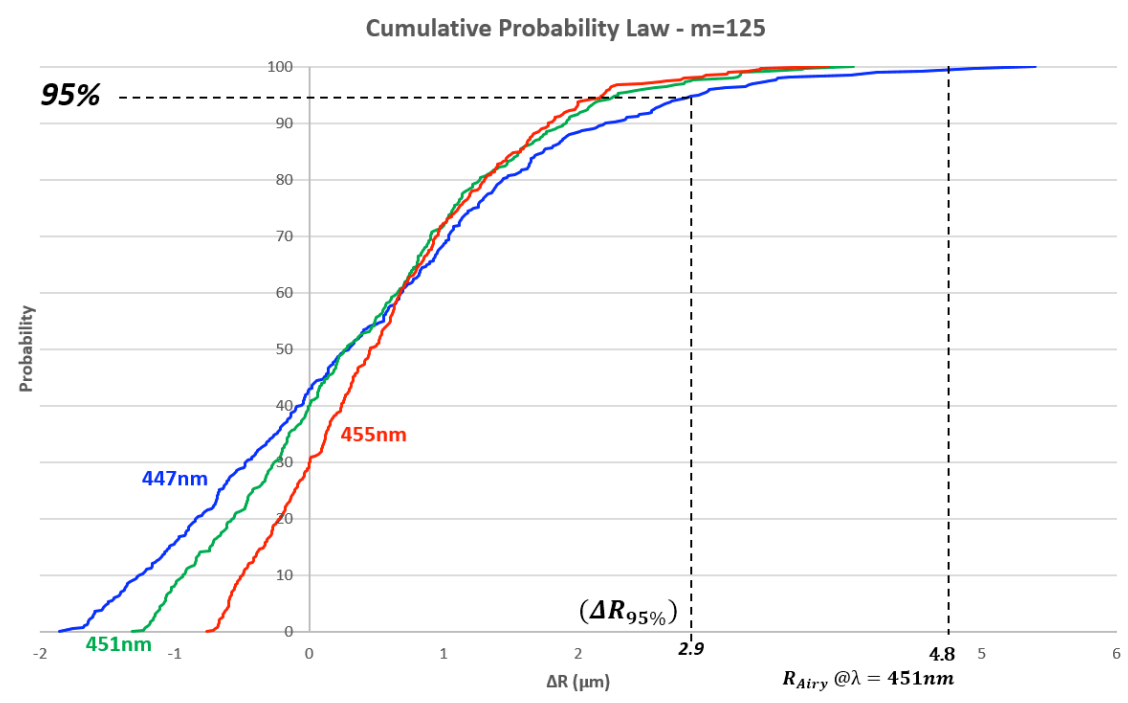

Figure 8. Cumulative probability for RMS spot radius based on our tolerancing analysis of assembly errors.

\subsection{Alignment errors}

We performed a tolerance analysis to estimate the spot radius enlargement $\Delta \mathrm{R}_{\text {Align }}$ due to the position and orientation errors of the optical components after alignment: the collimator, prism, grating and camera. We assumed that i) the maximum position error will be equal to $+/-100 \mu \mathrm{m}$ in every direction $\mathrm{x}, \mathrm{y}, \mathrm{z}$ and ii) the maximum collimator orientation errors will be equal to $+/-0.2$ degree around the $\mathrm{x}$ and the $\mathrm{y}$ axes. We have assumed that the maximum error on the fiber positioning along the optical axis will be equal to $+/-250 \mu \mathrm{m}$. The tolerancing analysis comprised 540 runs that corresponds to approximatively 20 runs per tolerancing parameter. We have included the following compensators: detector focus and tip-tilt.

The results show that in the realistic case, the maximum spot image radius variation is $\Delta \mathrm{R}_{\mathrm{Align}} \approx 2.4 \mu \mathrm{m}$ which is reached at the diffraction order $\mathrm{m}=141$ for the wavelength $\lambda_{\mathrm{Max}, 141}=405 \mathrm{~nm}$ (Figure 9). At the diffraction orders $\mathrm{m}=80$ and 106 , the realistic spot image radius variation is always lower than $1.2 \mu \mathrm{m}$. At the diffraction order $\mathrm{m}=125$, the maximum spot image radius variation is equal to $1.4 \mu \mathrm{m}$. 


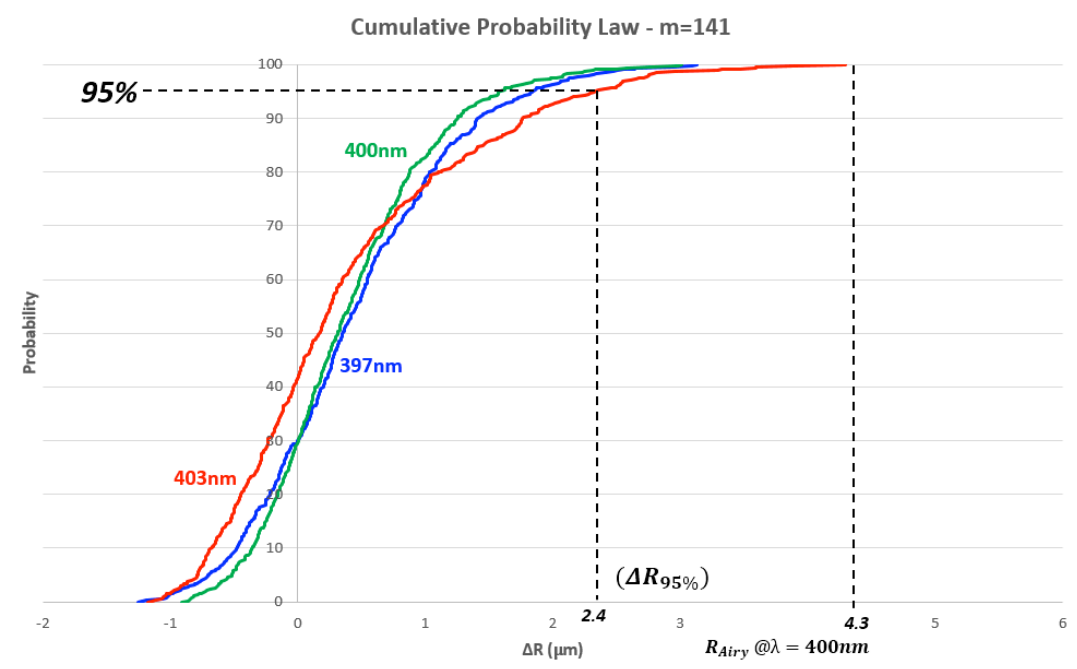

Figure 9. Cumulative probability for RMS spot radius based on our tolerancing analysis of assembly errors.

\subsection{Image spot radius performance budget}

We have assumed that spot image radius after manufacturing, assembly and alignment at the wavelength $\lambda \mathrm{j}, \mathrm{m}$ of the diffraction order $\mathrm{m}$ is:

$$
R\left(\lambda_{j, m}\right)=R_{\text {Theoretical }}\left(\lambda_{j, m}\right)+\Delta R,
$$

where $R_{\text {Theoretical }}$ is the RMS spot size of the theoretical system in which the optical surfaces and the position of the optical components are perfect. $\Delta \mathrm{R}$ is the spot image radius enlargement due to the manufacturing, assembly and alignment errors calculated as follows

$$
\Delta R=\sqrt{\Delta R_{\text {Manufacturing }}\left(\lambda_{j, m}\right)^{2}+\Delta R_{\text {Assy }}\left(\lambda_{j, m}\right)^{2}+\Delta R_{\text {Align }}\left(\lambda_{j, m}\right)^{2}} .
$$

We have represented in Tables 4, 5, 6 and 7 the values of $\Delta \mathrm{R}_{\text {Manufacturing, }} \Delta \mathrm{R}_{\text {Assy }}, \Delta \mathrm{R}_{\text {Align }}$ and $\Delta \mathrm{R}$ at the diffraction orders $m$ $=80,106,125$ and 141 respectively thus covering the specified spectral region. We notice that the major contributors to the spot size enlargement are the assembly errors at the diffraction orders $m=80,106$ and 125 . At the diffraction order $m$ $=141$ for the wavelengths comprised between $397 \mathrm{~nm}$ and $403 \mathrm{~nm}$, the major contributors to the performance are mostly the assembly and the alignment errors.

Over the specified spectral region, we estimate that the minimum spot image radius enlargement is close to $2 \mu \mathrm{m}$ and is reached at the diffraction order $m=80$. At the diffraction orders $m=106,125$ and 141 the enlargement of the spot radius is close to $3 \mu \mathrm{m}$ and reaches a maximum equal to $3.6 \mu \mathrm{m}$ at $\lambda_{\min , 125}=447 \mathrm{~nm}$.

Table 4. Results of the spot image radius tolerancing analysis for the realistic case of the fabrication, assembly and alignment errors at diffraction order $m=80$.

\begin{tabular}{cccc}
\hline Contributor & $\lambda_{\min , \mathbf{8 0}}=\mathbf{7 0 0} \mathbf{~ n m}$ & $\lambda_{\mathbf{C}, 80}=\mathbf{7 0 5} \mathbf{~ n m}$ & $\lambda_{\text {Max }, 80}=\mathbf{7 1 0} \mathbf{n m}$. \\
\hline$\Delta \mathbf{R}_{\text {Manufacturing }}$ & $1.1 \mu \mathrm{m}$ & $1.0 \mu \mathrm{m}$ & $1.0 \mu \mathrm{m}$ \\
$\Delta \mathbf{R}_{\text {Assy }}$ & $1.6 \mu \mathrm{m}$ & $1.6 \mu \mathrm{m}$ & $1.6 \mu \mathrm{m}$ \\
$\Delta \mathbf{R}_{\text {Alignment }}$ & $0.7 \mu \mathrm{m}$ & $0.7 \mu \mathrm{m}$ & $0.9 \mu \mathrm{m}$ \\
\hline$\Delta \mathbf{R}$ (RSS) & $\mathbf{2 . 1} \boldsymbol{\mu m}$ & $\mathbf{2 . 0} \boldsymbol{\mu m}$ & $\mathbf{2 . 1} \boldsymbol{\mu m}$ \\
\hline
\end{tabular}

Table 5. Results of the spot image radius tolerancing analysis for the realistic case of the fabrication, assembly and alignment errors at diffraction order $m=106$. 


\begin{tabular}{cccc}
\hline Contributor & $\lambda_{\text {min, } 106}=\mathbf{5 2 8} \mathbf{~ n m}$ & $\lambda_{\mathrm{C}, \mathbf{1 0 6}}=\mathbf{5 3 2} \mathbf{~ n m}$ & $\lambda_{\text {Max }, 106}=\mathbf{5 3 6} \mathbf{n m}$. \\
\hline$\Delta \mathbf{R}_{\text {Manufacturing }}$ & $1.5 \mu \mathrm{m}$ & $1.3 \mu \mathrm{m}$ & $1.1 \mu \mathrm{m}$ \\
$\Delta \mathbf{R}_{\text {Assy }}$ & $2.3 \mu \mathrm{m}$ & $2.1 \mu \mathrm{m}$ & $2.1 \mu \mathrm{m}$ \\
$\Delta \mathbf{R}_{\text {Alignment }}$ & $0.7 \mu \mathrm{m}$ & $0.9 \mu \mathrm{m}$ & $1.2 \mu \mathrm{m}$ \\
\hline$\Delta \mathbf{R}$ (RSS) & $\mathbf{2 . 8} \boldsymbol{\mu m}$ & $\mathbf{2 . 6} \boldsymbol{\mu m}$ & $\mathbf{2 . 1} \boldsymbol{\mu m}$ \\
\hline
\end{tabular}

Table 6. Results of the spot image radius tolerancing analysis for the realistic case of the fabrication, assembly and alignment errors at diffraction order $m=125$

\begin{tabular}{cccc}
\hline Contributor & $\lambda_{\text {min, }, 125}=\mathbf{4 4 7} \mathbf{~ n m}$ & $\lambda_{\mathrm{C}, 125}=\mathbf{4 5 1} \mathbf{~ n m}$ & $\lambda_{\text {Max }, 125}=\mathbf{4 5 5} \mathbf{~ n m . ~}$ \\
\hline$\Delta \mathbf{R}_{\text {Manufacturing }}$ & $1.9 \mu \mathrm{m}$ & $1.5 \mu \mathrm{m}$ & $1.3 \mu \mathrm{m}$ \\
$\Delta \mathbf{R}_{\text {Assy }}$ & $2.9 \mu \mathrm{m}$ & $2.3 \mu \mathrm{m}$ & $2.2 \mu \mathrm{m}$ \\
$\Delta \mathbf{R}_{\text {Alignment }}$ & $0.9 \mu \mathrm{m}$ & $1 \mu \mathrm{m}$ & $1.4 \mu \mathrm{m}$ \\
\hline$\Delta \mathbf{R}$ (RSS) & $\mathbf{3 . 6} \boldsymbol{\mu m}$ & $\mathbf{2 . 9} \boldsymbol{m}$ & $\mathbf{2 . 9} \boldsymbol{\mu m}$ \\
\hline
\end{tabular}

Table 7. Results of the spot image radius tolerancing analysis for the realistic case of the fabrication, assembly and alignment errors at diffraction order $m=141$.

\begin{tabular}{|c|c|c|c|}
\hline Contributor & $\lambda_{\min , 141}=397 \mathrm{~nm}$ & $\lambda_{\mathrm{C}, 141}=400 \mathrm{~nm}$ & $\lambda_{\text {Max }, 141}=403 \mathrm{~nm}$. \\
\hline$\Delta \mathbf{R}_{\text {Manufacturing }}$ & $1.4 \mu \mathrm{m}$ & $1.2 \mu \mathrm{m}$ & $1.3 \mu \mathrm{m}$ \\
\hline$\Delta \mathbf{R}_{\text {Assy }}$ & $1.5 \mu \mathrm{m}$ & $1.7 \mu \mathrm{m}$ & $1.5 \mu \mathrm{m}$ \\
\hline$\Delta \mathbf{R}_{\text {Alignment }}$ & $1.9 \mu \mathrm{m}$ & $1.6 \mu \mathrm{m}$ & $2.4 \mu \mathrm{m}$ \\
\hline$\Delta \mathrm{R}(\mathrm{RSS})$ & $2.8 \mu \mathrm{m}$ & $2.6 \mu \mathrm{m}$ & $3.1 \mu \mathrm{m}$ \\
\hline
\end{tabular}

Figure 10 shows the value $R_{\text {Realisitc }}$ of the spot image radius after manufacturing, assembly and alignment for the minimum, central and maximum wavelengths of the spectral orders $m=80,106,125$ and 141 . We also represent the values of $R_{\text {Theoretical }}$ and of the Airy radii.

We notice that at the diffraction order $m=80$ for wavelengths between 700 and $710 \mathrm{~nm}, R_{\text {Realisitc }}$ is always lower than the Airy radii and that the system is diffraction limited. At the order of diffraction $m=106$, the system can still be considered as diffraction limited at wavelengths longer that $532 \mathrm{~nm}$ even if the image quality is slightly above the diffraction limit at a wavelength of $528 \mathrm{~nm}$.

The system is not any longer limited by diffraction at the diffraction orders $m=125$ and 141 of wavelength ranges 447 to

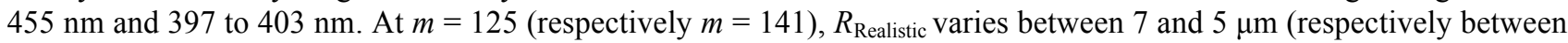
8 and $6 \mu \mathrm{m})$.

We deduced that in realistic conditions the spectrograph should be able to provide a spot radius close to the diffraction limit and smaller than $6 \mu \mathrm{m}$ rms over the spectral interval from 450 to $700 \mathrm{~nm}$. Over the spectral domain from 400 to $450 \mathrm{~nm}$, the spot image spot radius should be smaller than $8 \mu \mathrm{m}$. We also concluded that the image quality of this spectrometer is compatible with fiber of a maximum core diameter equal to $5 \mu \mathrm{m}$. 

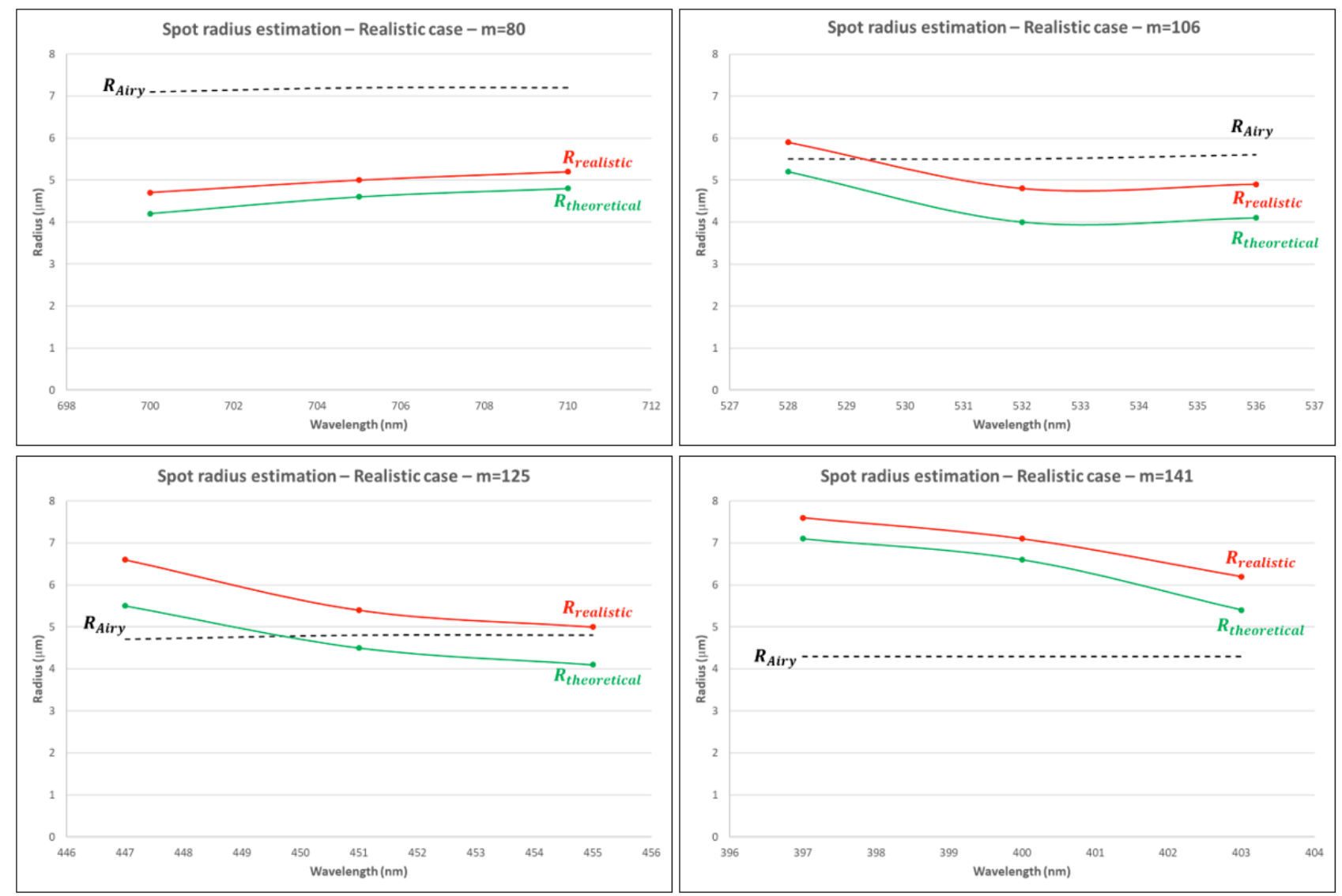

Figure 10. The cumulative probability of RMS spot sizes including manufacturing errors and tolerancing analysis.

\section{STRAY LIGHT ANALYSIS}

\subsection{Method and assumptions}

We have developed a non-sequential model of the EXOhSPEC spectrograph (Figure 11) to estimate the amount of stray light reaching the detector over the specified spectral region. The fiber optics output face is represented by one point-size source emitting a beam of cone semi-angle equal to 4 degrees at the following diffraction orders $m$ and wavelengths that covers the specified spectral domain:

- $\quad m=141: \lambda_{1,141}=397 \mathrm{~nm}, \lambda_{2,141}=399 \mathrm{~nm}, \lambda_{3,141}=400 \mathrm{~nm}, \lambda_{2,141}=401 \mathrm{~nm}$ and $\lambda_{5,141}=403 \mathrm{~nm}$.

- $\quad m=125: \lambda_{1,125}=447 \mathrm{~nm}, \lambda_{2,125}=449 \mathrm{~nm}, \lambda_{3,125}=451 \mathrm{~nm}, \lambda_{2,125}=453 \mathrm{~nm}$ and $\lambda_{5,125}=455 \mathrm{~nm}$.

- $m=106: \lambda_{1,106}=528 \mathrm{~nm}, \lambda_{2,106}=530 \mathrm{~nm}, \lambda_{3,106}=532 \mathrm{~nm}, \lambda_{2,106}=534 \mathrm{~nm}$ and $\lambda_{5,106}=536 \mathrm{~nm}$.

- $m=80: \lambda_{1,80}=700 \mathrm{~nm}, \lambda_{2,80}=702 \mathrm{~nm}, \lambda_{3,80}=705 \mathrm{~nm}, \lambda_{2,80}=707 \mathrm{~nm}$ and $\lambda_{5,80}=710 \mathrm{~nm}$.

We assumed that the faces of L1, L2 and L3 and of the dispersive prism are coated by one Anti-Reflective (AR) coating of reflectivity equal to $1 \%$. This corresponds to the reflectivity of the AR coating specified in our order to the suppliers of the collimator lenses and of the dispersive prism. We also assumed that the diffraction efficiency of the grating is equal to $50 \%$ and that folding mirror reflects $100 \%$ of the incident light. 


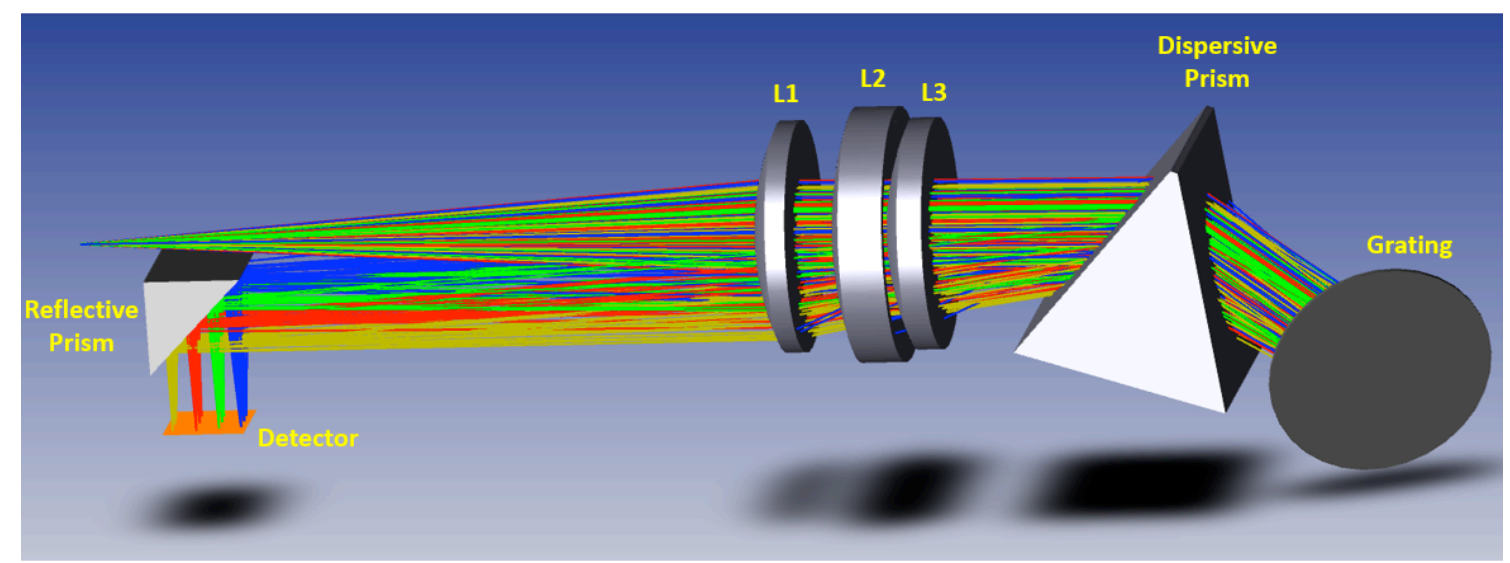

Figure 11. The optical beam is traced using a ZEMAX EXOhSPEC non-sequential model for the diffraction orders $m=80$, 106,125 and 141 (five wavelengths for each diffraction order) thus covering the specified spectral band from 400 to 700 nm.

\subsection{Results}

We have represented in Figure 12 the distribution of the irradiance on the detector. We notice that the stray light is mostly concentrated close to the diffraction orders $\mathrm{m}=80$ and 106 at wavelengths between 530 and $700 \mathrm{~nm}$. We have identified that the most important contributor to the straylight performance is the residual light reflected by the collimator anti-reflective coatings toward the reflective prism and the detector.

Figure 13 represents how the irradiance cross-section changes across the central detector column. The ratio between the science beam and the ghost image irradiance varies between 4,500 for the spectral orders lower than 106 to 20,000 for the spectral orders higher than 106 . We thus estimate that the maximum level stray light should be close to $0.1 \%$ of the scientific signal. This value is considered as acceptable when compared to HARPS where the amount of stray light level reaching the detector is equal to a few percent of the scientific signal [3] .

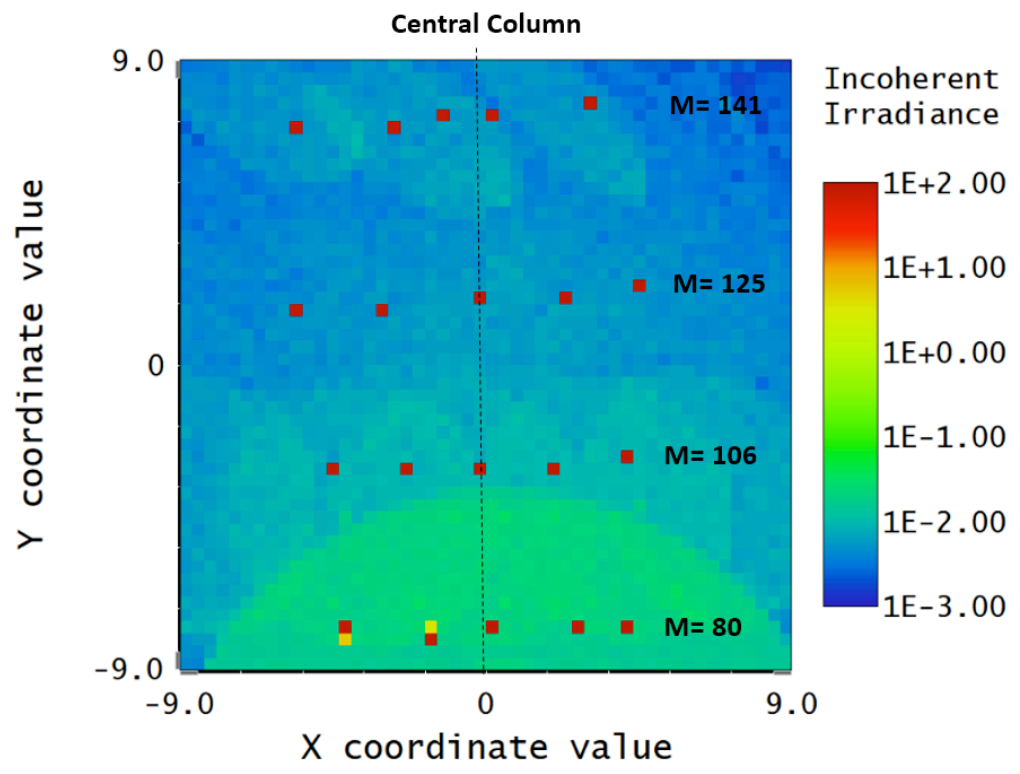

Figure 12. ZEMAX Detector view after stray light analysis ray for the diffraction orders $m=80,106,125$ and 144 . 


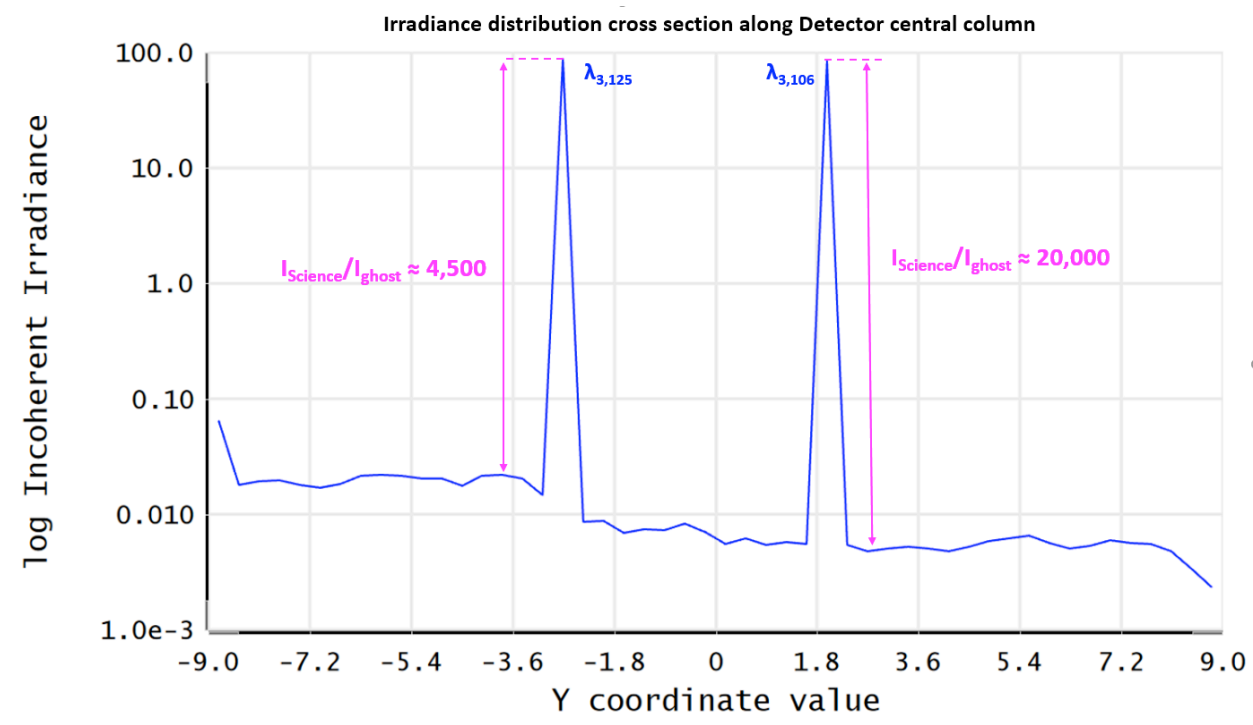

Figure 13. ZEMAX detector view of the cross-section along the central column.

\section{PROTOTYPE PRELIMINARY TESTS AND FUTURE DEVELOPMENTS}

\subsection{Prototype development at NARIT}

We have developed at NARIT a preliminary version of the EXOhSPEC prototype to test the overall systems by using off-the-shelf components only (Figure 14-left panel). The objective has been to verify that i) the prototype is able to provide spectra with a spectral resolution of at least 20,000 and ii) the processing algorithm developed by UH and used to extract the spectrum form the images provided by the camera is working. The prototype was installed on the dome of the $0.7 \mathrm{~m}$ Corrected Dall-Kirkham telescope manufactured by the company Plane Wave and located in the NARIT Astropark (Figure 14 middle and left panels). This prototype includes the following components:

- Injection unit equipped with a guiding camera for the company Sheylack [8] used to i) inject the light from the telescope on the fiber input face and ii) to guide the telescope to keep the star light focused on the fiber.

- Fiber of $50 \mu \mathrm{m}$ core diameter and of $20 \mathrm{~m}$ length used to connect the telescope to the spectrograph.

- Thorlabs achromatic lens of $50.8 \mathrm{~mm}$ diameter, focal length equal to $150 \mathrm{~mm}$ and anti-reflective coating optimized for the spectral region 650 to $1050 \mathrm{~nm}$.

- Air Cooled MicroLine ML8051 camera manufactured by Finger Lakes Instrumentation that comprises 3296 x 2472 pixels of size equal to $5.5 \mu \mathrm{m}$.

- One F2 equilateral dispersing prism from Thorlabs (model PS854) of length equal to $50 \mathrm{~mm}$.

Figure 15 represents the processed spectrum of a Thorium-Argon lamp and Figure 16 shows the 2D image of the spectrum of Sirius. This result show that the proposed prototype connected to a medium size telescope is already able to provide spectrum of a real star. We estimate from the data we have acquired that the spectral range of the spectrograph is 400 to $700 \mathrm{~m}$ and the spectral resolution is higher than 20,000 . 

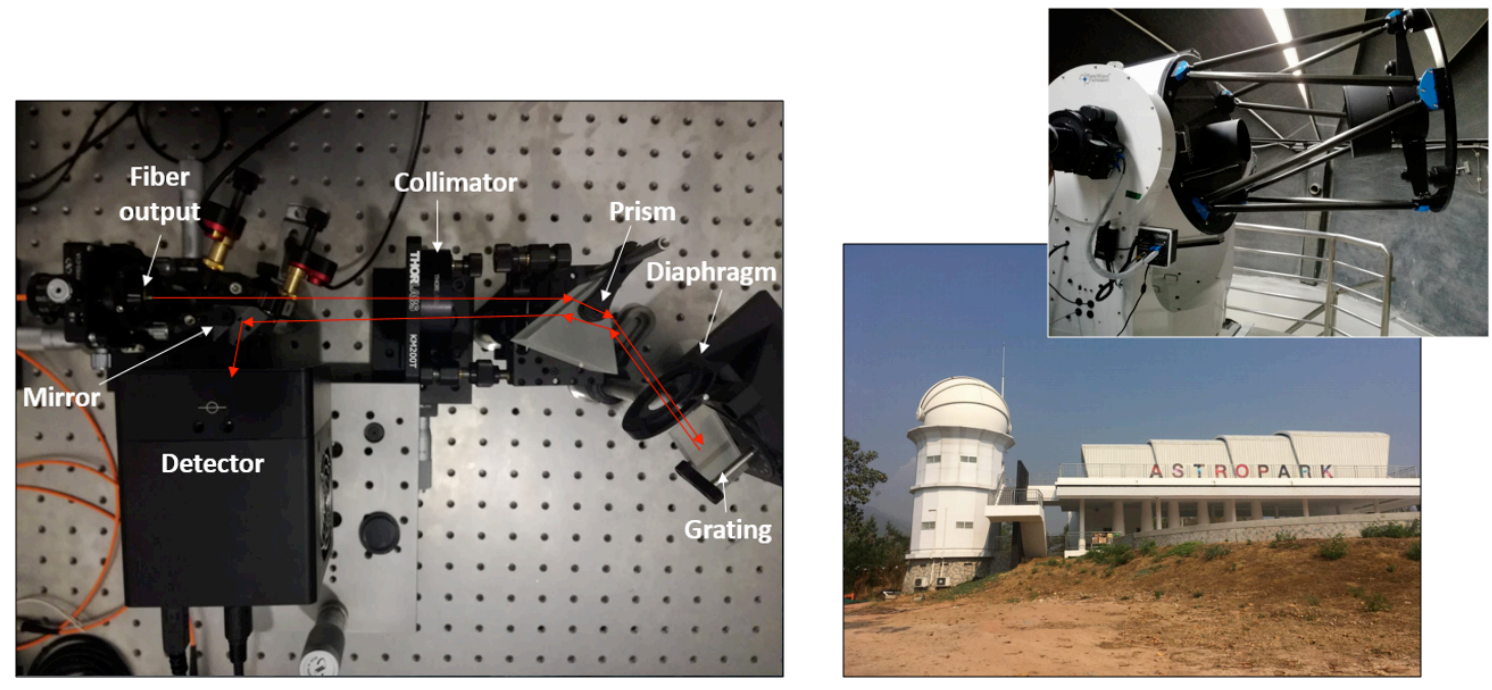

Figure 14. Preliminary EXOhSPEC prototype deployed inside NARIT's Astropark $0.7 \mathrm{~m}$ telescope dome (Chiang Mai, Thailand).

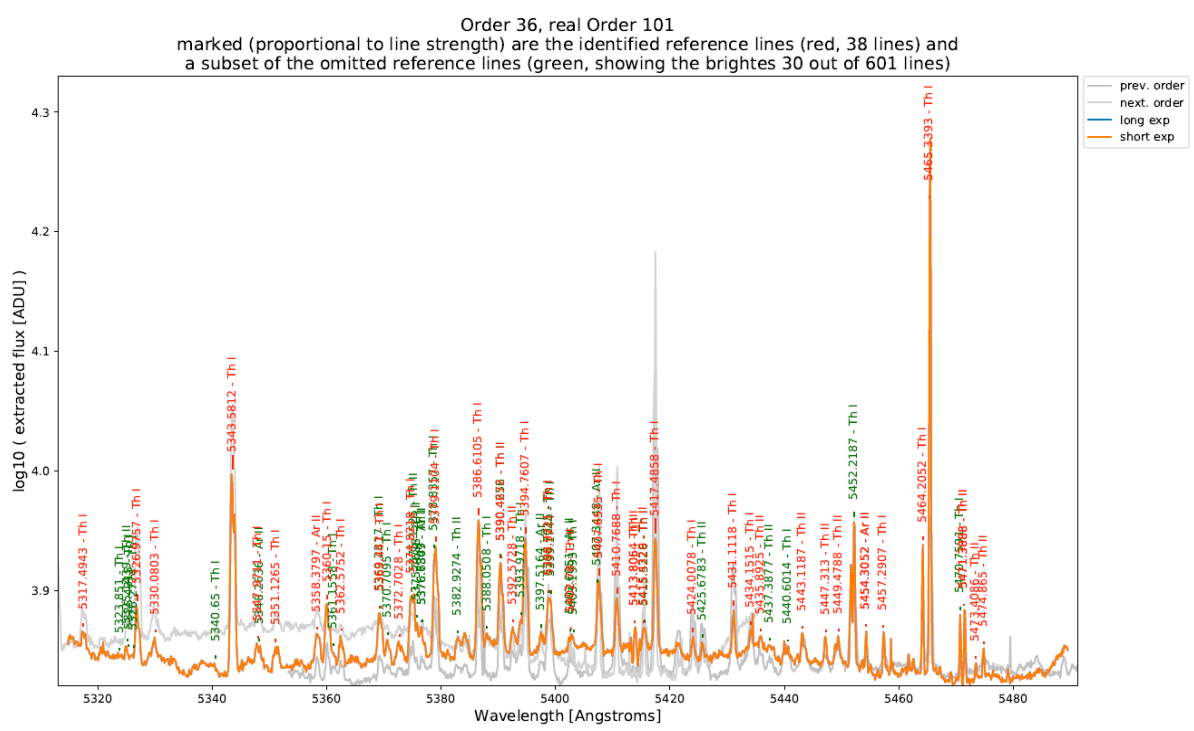

Figure 15. Example of the wavelength solution between 532 and $548 \mathrm{~nm}$. 
Figure 16. Image of the spectrum of Sirius acquired with the preliminary EXOhSPEC prototype connected to the $0.7 \mathrm{~m}$ telescope of NARIT Astropark .

\section{CONCLUSIONS}

We have presented the design and the performance estimation of the EXOhSPEC high resolution spectrograph. We showed that in operational conditions, the spectrograph should be able to provide images close to the diffraction limit over the spectral interval from 450 to $700 \mathrm{~nm}$. We presented the results of the stray light analyses and conclude that the stray light irradiance should be less than $0.1 \%$ of the scientific signal - a level considered as acceptable. Finally, we present the results obtained with the preliminary version of the prototype connected to the NARIT medium size telescope that comprise only off-the-shelf components. We showed that this spectrograph has been able to provide spectrum with a spectral resolution higher than 20,000 across the spectral range from 400 to $700 \mathrm{~nm}$.

The next steps will consist of i) manufacturing and testing an optimized collimator, ii) integrating the collimator into the spectrograph, iii) replacing the equilateral dispersive prism with a prism of angle equal to 50 degree and iv) replacing the air cooled camera by a scientific camera. The barrel of the collimator has already been designed and manufactured at NARIT [9]. We expect to integrate the lenses into the barrel and to measure the collimator wavefront quality in August 2019. The integration of this collimator, of the new prism and of the camera on EXOhSPEC will be performed in September and October 2019 with the objective to make the first on-sky observations with the TNT during November 2019.

\section{ACKNOWLEDGEMENT}

The University of Hertfordshire (UH) has been developing the EXOplanet high resolution SPECtrograph (EXOhSPEC) in the framework of the STFC Newton Fund (ST/R006598/1) in partnership with the National Astronomical Research Institute of Thailand (NARIT) and Queen Mary University of London.

\section{REFERENCES}

[1] F. Pepe, M. Mayor, B. Delabre, D. Kohler, D. Lacroix, D. Queloz, S. Udry, W. Benz, J.-L. Bertaux, J.-P. Sivan "HARPS: a new high-resolution spectrograph for the search of extrasolar planets", Proc. SPIE 4008, Optical and IR Telescope Instrumentation and Detectors, (2000);

[2] HARPS ESO website: https://www.eso.org/sci/facilities/lasilla/instruments/harps/inst/description.html (2019) 
[3] European Southern Observatory, HARPS User Manual, 3P6-MAN-ESO-90100-0005, Issue: 2.3, Date: February 1st, 2019

[4] C. Buisset; A. Prasit; A. Leckngam; T. Lépine; S. Poshyajinda; B. Soonthornthum; P. Irawati; A. Richichi; U. Sawangwit; V. Dhillon; L. K. Hardy, "Progress on the prevention of stray light and diffraction effects on the Thai National Telescope", SPIE 9626, Optical Systems Design 2015: Optical Design and Engineering VI, 96262E (2015);

[5] Thorlabs website (2019): https://www.thorlabs.com/newgrouppage9.cfm?objectgroup id=1124

[6] Optimax website (2019): https://www.optimaxsi.com/innovation/optical-manufacturing-tolerance-chart/

[7] C. Buisset, S. Poshyachinda, B. Soonthornthum, A. Prasit, M. A. Alagao, P. Choochalerm, W. Wanajaroen, T. Lepine, Y. Rabbia, S. Aukkaravittayapun, A. Leckngam, G. Thummasorn , S. Ngernsujja, A. Inpan, P. Kaewsamoet, E. Lhospice, P. Meemon, P. Artsang, K. Suwansukho, W. Sirichote, J. Paenoi "Activity status and future plans for the Optical Laboratory of the National Astronomical Research Institute of Thailand ", Proc. SPIE 10714, Third International Conference on Photonics Solutions (ICPS2017), 1071407 (2018)

[8] Sheylack Instrument website (2019): https://www.shelyak.com/

[9] Kawinkij, A. Prasit, C. Buisset, E. Lhospice, H. Jones, W. Martin, R. Errmann, P. Choochalerm, D. Campbell, S. Aukkaravittayapun, T. Panyaphirawat, S. Rattanasoon, A. Leckngam, D. Mkrtichian, C. Baker, G. AngladaEscude, "EXOhSPEC collimator mechanical design", SPIE Astronomical Optics: Design, Manufacture, and Test of Space and Ground Systems II , (2019) 